Research Article

\title{
Combined Fuzzy Clustering and Chan_Vese Model for Multicolor Gradual Ochotona curzoniae Image Segmentation
}

\author{
Haiyan Chen $(\mathbb{D})$ and Huaqing Zhang \\ School of Computer and Communication, Lanzhou University of Technology, Lanzhou, Gansu 730050, China \\ Correspondence should be addressed to Haiyan Chen; chenhaiyan@sina.com
}

Received 2 November 2020; Revised 21 January 2021; Accepted 31 January 2021; Published 16 February 2021

Academic Editor: Francisco Periago Esparza

Copyright (c) 2021 Haiyan Chen and Huaqing Zhang. This is an open access article distributed under the Creative Commons Attribution License, which permits unrestricted use, distribution, and reproduction in any medium, provided the original work is properly cited.

\begin{abstract}
Precise segmentation of Ochotona curzoniae images collected in a nature scene is the basis of Ochotona curzoniae recognition and behavior analysis. Ochotona curzoniae images have the characteristics of diversity and graduality of target colors and complex background. The method of combined Chan_Vese model and $k$-means clustering algorithm is used to segment the multicolor images, but when $k$-means clustering algorithm is used to cluster the color of multicolor images, the manner of hard classification is adopted, without considering the color-gradient feature. As a resolution to this problem, a new approach of the Chan_Vese model in combination with fuzzy $C$-means clustering is proposed in the present paper. The proposed model utilises fuzzy $C$-means clustering to cluster the pixels inside the evolution curve of the Chan_Vese model, classifying the pixels into a certain color cluster with a certain probability to describe the image color gradual characteristics. By fuzzy $C$-means clustering, several cluster centers can be obtained, and the values of cluster centers can be used to replace internal fitting values of the Chan_Vese model. In this way, the problem that the Chan_Vese model cannot segment images with intensity inhomogeneity is overcome. Furthermore, the global Heaviside function is replaced by the local Heaviside function to suppress the influence of the background on image segmentation. The experimental results of Ochotona curzoniae images segmentation demonstrate that the proposed model can more accurately locate the target contour and has a higher Dice similarity coefficient, Jaccard Similarity, and segmentation accuracy.
\end{abstract}

\section{Introduction}

Rodent damage is a significant threat to the environment of the Qinghai Tibet Plateau, where Ochotona curzoniae is the dominant rat species. The study of Ochotona curzoniae is conducive to the protection of the environment of the Qinghai Tibet Plateau, with precise segmentation of Ochotona curzoniae images being the basis of detection, recognition, and behavior analysis thereof [1]. Ochotona curzoniae images collected in the natural environment have a complex background, low contrast, and target with various and gradual color, exacerbating the difficulty of image segmentation. Traditional image segmentation methods have low accuracy and cannot completely and effectively segment the target in Ochotona curzoniae images [2]. Active contour models can effectively segment low-contrast and complex images by using image information, including intensity information and gradient information. The problem of topological structure changes of the evolution curve can also be effectively solved.

Active contour can be categorized into edge-based models and region-based models [3]. Region-based models are widely used and utilize the region information of the image to drive the motion of the topological structure of the evolution curve, thereby providing a good segmentation quality for weak-edge and noise images [4]. The Chan_Vese (CV) model is a classical region-based active contour model with good segmentation results in a weak-edge image [5]. However, the CV model was proposed for cases where the image color distribution is homogeneous; thus, the model cannot segment images with color diversity.

Numerous methods have been proposed to resolve this problem, including two classes: single model and hybrid model. Single model simply involves modification and 
deformation of the level set model. Li et al. proposed the local binary fitting model (LBF), which used the Gaussian kernel function to compute the intensity means of the local region. Here, the internal and external intensity means of the evolution curve in the CV model were replaced by the intensity mean of the local region, and the kernel function was introduced to fit the energy term of the energy function, thereby fitting the real intensity information of the image by using the local intensity information. Although the segmentation results of synthetic images and medical images have indicated that the method can better segment the intensity inhomogeneous image, the model can easily fall into a local minimum [6]. A local image fitting model (LIF) was suggested by Zhang et al., which extracted the local intensity information of the image through a truncated Gaussian kernel function and used the acquired local intensity information to construct a fitting image. The difference between the fitting image and the original image was used to fit the energy function. The segmentation performance of LIF for medical images and synthetic images was consistent with the LBF model and had reduced running times [7]. Ma et al. formulated an adaptive local fitting active contour model that took each pixel in the original image as the center, with a $5 \times 5$ rectangular window being constructed to limit the pixel region. The intensity mean of each region and the deviation value between the intensity mean and the original intensity value were calculated to construct a new pixel fitting value, while the deviation value was also used for constructing the kernel function. To establish a new energy function, the new pixel fitting value and the kernel function were used for replacing the pixel fitting value and kernel function of the LBF model. The segmentation results of medical images and synthetic images have depicted that the model can effectively segment intensity inhomogeneity images [8]. The inhomogeneous entropy descriptor and an entropy weighted energy function predicated on three different local fitted images were both introduced into the level set model by Wang et al. The model used the absolute value of the squared difference between the original image pixels and intensity mean to build an inhomogeneous entropy descriptor, with the square of the original image being used to construct extended fitting images according to the construction manner of the LIF model fitting image. Further, inhomogeneous entropy descriptors and extended fitting images were introduced into the LHIF model to construct new energy functions. The segmentation results in synthetic images, medical images, and natural images have indicated that the model is effective in segmenting images with intensity inhomogeneity [9]. Though the above methods can segment intensity inhomogeneity images, the segmentation results are sensitive to the initial contour.

Hybrid models combine other methods with the level set model, such as the clustering algorithm, to address the aforementioned problem. Filtering an original image by coherence-enhanced diffusion filter was first attempted by Marwa Braiki et al. After filtering, the $k$-means clustering algorithm ( $k$-means) was used for clustering the filtered image into 3 classes, and morphological filtering was used to clean up the binary mask; the result after cleaning was used as the initial contour of the active contour model. Finally, the $\mathrm{CV}$ model was used to segment images. The superiority of this method in segmenting weak edge images was demonstrated by the segmentation results for human dendritic cells [10]. Memari et al. employed an enhanced fuzzy $C$-means clustering algorithm by genetic algorithm to extract the contour of an area of interest in the preprocessed retinal blood vessel image; the contour being used as the initial contour of the level set. In this method, the level set model was used in the end to segment the retinal blood vessel image. The segmentation experiments on the retinal blood vessel image have exhibited that the model can effectively segment images with low contrast and noise [11]. Jin et al. adopted an improved fuzzy $C$-means clustering algorithm to divide the image pixels into two categories. The two clustering centers construct an adaptive function to replace the weighting coefficient of the area term in the distance regularized level set evolution (DRLSE) model. Furthermore, novel edge detection operators and double-well potential functions were constructed to replace the edge detection operators and double-well potential functions in the DRLSE model. The segmentation of synthetic images, medical images, and animal and plant images has demonstrated that the model can quickly and accurately segment images with intensity inhomogeneity [12]. Fuzzy $C$-means clustering was applied by Bin Dong et al. to cluster images. Here, the cluster center values were utilized as the deviation field estimation, the deviation field was introduced into the bias correction active contour model to construct the energy function, a novel distance regular term was constructed and added into the energy function to construct a novel energy function of the active contour model, and the energy function was minimized by improving the method. Segmentation experiments on synthetic images and medical images have revealed that the model is able to effectively segment images with intensity inhomogeneity [13]. The aforementioned models can effectively segment intensity inhomogeneity images and are not sensitive to the initial contour, but at the same time studies on multicolor color-gradient image segmentation are scarce.

In terms of segmenting the multicolor images, Zhang et al. proposed a $k$-means CV model. The model introduced the $k$-means clustering algorithm into the CV model. Several target color classes were obtained by $k$-means clustering to describe the multicolor characteristics of the target. The inner fitting value of the CV model was constructed by the clustering center values and mean filtering image. The segmentation results on the Ochotona curzoniae images have demonstrated that the method can effectively segment multicolor images [14]. Yet, this method used the $k$-means clustering algorithm to cluster the color of an object under the assumption of color mutation. Here, $k$-means clustering belonged to hard clustering, and all pixels were classified into a certain color cluster by hard classification without considering the color graduality of images in the clustering process.

To address the problem, the fuzzy $C$-means clustering algorithm was used in the present study to cluster the intensity values within the evolution curve, and these pixels 
were classified into different color clusters with a certain probability, so as to allow for adaption to the image color graduality, and thus achieve enhancement of the fit and improvement of the internal fitting values accuracy in the CV model.

The rest of this paper is arranged as follows: in Section 2, the proposed model is described in detail; the experiments and analysis between the proposed model and other models are discussed in Section 3; and Section 4 is the summary of the present paper.

\section{The Proposed Model}

The CV model [5] has been extensively used for image segmentation and drives curve evolution through the difference of intensity information in different regions of images; the energy function of the $\mathrm{CV}$ model was exhibited therein.

$$
F\left(c_{1}, c_{2}, \varphi\right)=\mu \int_{\Omega} \delta_{\varepsilon}(\varphi) \cdot|\nabla \varphi(x, y)| \mathrm{d} x \mathrm{~d} y+\lambda_{1} \int_{o(C)}\left|I(x, y)-c_{1}\right|^{2} H_{\varepsilon}(\varphi(x, y)) \mathrm{d} x \mathrm{~d} y+\lambda_{2} \int_{i(C)}\left|I(x, y)-c_{2}\right|^{2}\left|I(x, y)-c_{2}\right|^{2}
$$

where $\mu, \lambda_{1}$, and $\lambda_{2}$ are the positive weight parameters. Curve $C$ is the evolution curve. Constants $c_{1}$ and $c_{2}$ are the intensity mean outside and inside of curve $C$, respectively, as denoted in equation (2). $\varphi(x, y)$ is the level set function. $o(C)$ and $i(C)$ indicate the outer and inner regions of curve $C$, respectively. $H_{\varepsilon}(\varphi(x, y))$ is the Heaviside function, as presented in equation (3), and $\delta_{\varepsilon}(\varphi(x, y))$ is the Dirac function, as depicted in equation (4). $\Omega$ is the image region and $\nabla$ is a gradient operator.

$$
\begin{aligned}
& c_{1}=\frac{\int_{\Omega} I(x, y) H_{\varepsilon}(\varphi(x, y)) \mathrm{d} x \mathrm{~d} y}{\int_{\Omega} H_{\varepsilon}(\varphi(x, y)) \mathrm{d} x \mathrm{~d} y}, \\
& c_{2}=\frac{\int_{\Omega} I(x, y)\left(1-H_{\varepsilon}(\varphi(x, y))\right) \mathrm{d} x \mathrm{~d} y}{\int_{\Omega}\left(1-H_{\varepsilon}(\varphi(x, y))\right) \mathrm{d} x \mathrm{~d} y}, \\
& H_{\varepsilon}(\varphi(x, y))=\frac{1}{2}\left(1+\frac{2}{\pi} \arctan \left(\frac{\varphi}{\varepsilon}\right)\right), \\
& \delta_{\varepsilon}(\varphi(x, y))=\frac{\varepsilon}{\pi\left(\varepsilon^{2}+\varphi^{2}\right)} .
\end{aligned}
$$

In equations (3) and (4), $\varepsilon$ is the regularization constant, which can control how fast the function $H_{\varepsilon}(\cdot)$ rises from 0 to 1 and the effective width of the function $\delta_{\varepsilon}(\cdot)$, so as to control the evolution speed of the model.

In the $\mathrm{CV}$ model, $c_{1}$ and $c_{2}$ are single variables and do not efficiently represent the features of diversity and graduality of target colors. Thus, the CV model cannot segment images that have the features of a complex background as well as diversity and graduality of the target colors.

In resolving the aforementioned problems, a new internal composite value that contains more image information was constructed in the present study. Meanwhile, a new external fitting value was constructed that can substantially reduce the influence of the background on image segmentation.

Firstly, fuzzy $C$-means clustering was used in the present study to cluster the pixels inside the evolution curve of the
CV model, and the pixels were divided into a certain pixel cluster on the basis of a certain probability to reflect the image color graduality characteristics. The internal fitting values of the $\mathrm{CV}$ model were replaced by the obtained several cluster centroid values. Simultaneously, the intensity values between the cluster centroid values and isolated background points were considerably similar because of the low contrast; thus, the dependence of the proposed model on the clustering results was reduced by adding mean-filtered images. In accordance with the above, the improved internal fitting value is shown in the following equation:

$$
c_{2 i}(x, y)=\alpha v_{i}+(1-\alpha) \operatorname{ave}(I)
$$

where $\alpha(1>\alpha>0)$ is a weight parameter, $v_{i}$ is the cluster center value of the $i^{\text {th }}$ cluster, and ave $(I)$ is the image after mean filtering.

Fuzzy $C$-means clustering is a soft clustering with a membership for each sample $[15,16]$. The objective function of fuzzy $C$-means clustering is depicted in the following equation:

$$
J(\mathbf{U}, \mathbf{K})=\sum_{i=1}^{c} \sum_{j=1}^{n} u_{i j}^{m}\left\|x_{j}-v_{i}\right\|^{2}
$$

where $\mathbf{U}$ represents a fuzzy matrix of $c \times n . \mathbf{K}=\left[v_{1}, \ldots, v_{c}\right]$ is the cluster center vector set. $v_{i}$ is the cluster center value of $i^{\text {th }}$ cluster and $c$ is the number of clusters. $n$ indicates the number of samples. $u_{i j}$ is the membership function, representing the probability that the $j^{\text {th }}$ sample belongs to the $i^{\text {th }}$ cluster. $u_{i j}$ satisfies the conditions of (7)

$$
\begin{aligned}
& \sum_{i=1}^{c} u_{i j}=1, \quad j=1,2, \ldots, n, \\
& u_{i j} \geq 0, \quad i=1,2, \ldots, c, j=1,2, \ldots, n .
\end{aligned}
$$

Fuzzy $C$-means clustering continuously updates the cluster center value and membership function until achieving optimal values. The updated process is exhibited in 


$$
\begin{aligned}
v_{i} & =\frac{\sum_{j=1}^{n} u_{i j}^{m} x_{j}}{\sum_{j=1}^{n} u_{i j}^{m}}, \quad i=1,2, \ldots, c, \\
u_{i j} & =\frac{1}{\sum_{n=1}^{c}\left[\left\|\left(x_{j}-v_{i}\right) /\left(x_{j}-v_{n}\right)\right\|\right]^{2 /(m-1)}}, \quad i=1,2, \ldots, c, j=1,2, \ldots, n .
\end{aligned}
$$

When fuzzy $C$-means clustering is employed to cluster the pixels of multicolors and color-gradient images, all pixels in an image are divided into color clusters with a certain probability [17]. This classification manner is appropriate for the characteristics of color graduality.

The internal fitting energy function is denoted in

$$
F_{i(C)}=\int_{i(C)}\left|I(x, y)-c_{2 i}\right|^{2}\left(1-H_{\varepsilon}(\varphi(x, y))\right) \mathrm{d} x \mathrm{~d} y .
$$

The novel internal fitting values contain richer image intensity information in equation (11). The influences of isolated background points on the clustering results are reduced by mean filtering. Here, the novel internal fitting values can wholly represent the intensity information inside the initial contour of the image and better adapt to the features of image color graduality.

Secondly, the local Heaviside function is used to construct the external fitting value of the $\mathrm{CV}$ model so as to suppress the influence of the background on image segmentation. The local Heaviside function is as denoted in

$$
H^{\prime}(x)= \begin{cases}0, & (x<-1), \\ \frac{x^{2}}{2}+x+\frac{1}{2}, & (-1 \leq x<0), \\ \frac{-x^{2}}{2}+x+\frac{1}{2}, & (0 \leq x<1) \\ 1, & (x \geq 1) .\end{cases}
$$

Hence, the external fitting value can be calculated as revealed in

$$
c_{1}^{\prime}=\frac{\int_{\Omega} I(x, y) H^{\prime}(\varphi(x, y)) \mathrm{d} x \mathrm{~d} y}{\int_{\Omega} H^{\prime}(\varphi(x, y)) \mathrm{d} x \mathrm{~d} y} .
$$

The external fitting energy function of the improved CV model is denoted in

$$
F_{o(C)}=\int_{o(C)}\left|I(x, y)-c_{1}^{\prime}\right|^{2} H^{\prime}(\varphi(x, y)) \mathrm{d} x \mathrm{~d} y .
$$

In equation (12), the proposed model restricts the search range through the local Heaviside function, effectively restraining the effect of the background on segmentation.

The improved energy function is shown in

$$
\begin{aligned}
F\left(c_{1}^{\prime}, c_{2 i}, \varphi\right)= & \mu \int_{\Omega} \delta^{\prime}(\varphi(x, y)) \cdot|\nabla \varphi(x, y)| \mathrm{d} x \mathrm{~d} y+\lambda_{1} F_{o(C)}+\lambda_{2} F_{i(C)} \\
= & \mu \int_{\Omega} \delta^{\prime}(\varphi(x, y)) \cdot|\nabla \varphi(x, y)| \mathrm{d} x \mathrm{~d} y+\lambda_{1} \int_{o(C)}\left|I(x, y)-c_{1}^{\prime}\right|^{2} H^{\prime}(\varphi(x, y)) \mathrm{d} x \mathrm{~d} y \\
& +\lambda_{2} \int_{i(C)}\left|I(x, y)-c_{2 i}\right|^{2}\left(1-H^{\prime}(\varphi(x, y))\right) \mathrm{d} x \mathrm{~d} y .
\end{aligned}
$$

In equation (15), $\delta^{\prime}(\varphi(x, y))$ is the derivative of the function $H^{\prime}(\varphi(x, y))$.
The evolution equation for the improved CV model is obtained by gradient descent, as shown in the following equation:

$$
\frac{\partial \varphi}{\partial t}=\delta^{\prime}(\varphi)\left[\mu \operatorname{div}\left(\frac{\nabla \varphi}{|\nabla \varphi|}\right)-\lambda_{1}\left(I(x, y)-c_{1}^{\prime}\right)^{2}+\lambda_{2}\left(I(x, y)-c_{2 i}(x, y)\right)^{2}\right]
$$

where div is the divergence operator, $\operatorname{div}(\nabla \varphi /|\nabla \varphi|)$ is the curvature of the evolution curve. When the value of energy function is minimum, the curve evolution stops and the segmentation ends.
The updated equation for the CV model is shown in

$$
\varphi^{n+1}=\varphi^{n}+\frac{\partial \varphi}{\partial t} \mathrm{~d} t
$$


where $\varphi^{n+1}$ is the level set function after $n+1$ iterations, $\varphi^{n}$ is the level set function afterniterations, and $\mathrm{d} t$ is the time step.

With regard to reducing the time complexity of the proposed model, the proposed model only clusters once for the intensity value of the image within the initial contour, and the subsequent evolution of the proposed model follows the result of the first clustering.

The algorithm flow of the proposed model is exhibited in Algorithm 1.

\section{Experiment and Analysis}

A group of Ochotona curzoniae images collected from Qinghai Tibet Plateau (East longitude 1013 ${ }^{\circ} \circ 5^{\prime} 36^{\prime \prime}$ $\sim 102^{\circ} 58^{\prime} 15^{\prime \prime}$, North latitude $33^{\circ} 58^{\prime} 21^{\prime \prime} \sim 34^{\circ} 48^{\prime} 48^{\prime \prime}$ ) was selected as experimental data (total 30 pieces). The image size was set as $256 \mathrm{pi} \times 256 \mathrm{pi}$. The selected parameters make the model optimal, which were set as follows: $c=10, \alpha=0.8$, $\lambda_{1}=\lambda_{2}=1$, and $\mu=0.02 * 255 * 255$.

To verify the segmentation effect of the proposed model, six classical active contour models were used for comparison and analysis with the proposed model, including CV, LBF, LIF, LGIF, Ding's, and Zhang's [5-7, 14, 18, 19]. Said models and the proposed model were used to segment 30 Ochotona curzoniae images. In the present study, the parameters of the CV model were $\mu=0.02 * 255 * 255, \lambda_{1}=\lambda_{2}=0.5, \varepsilon=1$, and $v=0$, the parameters of the LBF model were $v=0.02 * 255 * 255, \lambda_{1}=\lambda_{2}=0.5, \varepsilon=1, \mu=1$, and $\sigma=3$, the parameters of the LIF model were $\varepsilon=1.5, \sigma=3$, the parameters of the LGIF model were $v=0.02 * 255 * 255$, $\lambda_{1}=\lambda_{2}=0.5, \varepsilon=1, \mu=1$, and $w=0.05$, the parameters of Ding's model were $\mu=0.002 * 255 * 255, \lambda_{1}=\lambda_{2}=1, \varepsilon=1$, $\mu=1, \sigma=0.05$, and $\Delta=20$, and the parameters of Zhang's model were $k=10, \quad \alpha=0.8, \quad \lambda_{1}=\lambda_{2}=1, \quad$ and $\mu=0.02 * 255 * 255$. The segmentation results of six images were randomly selected for analysis, as displayed in Figure 1.

As observed in Figure 1, the first row is the original image with the initial contour of the target, and the second to last rows contain the segmentation results of the $\mathrm{CV}$ model, LBF model, LGIF model, Ding's model, Zhang's model, the proposed model, and ground truth images, respectively. An observation can also be made that the Ochotona curzoniae images have the characteristics of low contrast and target color with diversity and graduality. As against the models of CV, LBF, LIF, LGIF, and Ding, the segmentation results of the proposed model demonstrate a substantial improvement was achieved. Meanwhile, the proposed model also demonstrated better performance when compared with Zhang's model. Since the proposed model uses fuzzy $C$-means clustering to cluster the pixels inside the initial contour of the $\mathrm{CV}$ model, and the pixels inside the initial contour of the $\mathrm{CV}$ model are divided into a certain color cluster with a certain probability, both the color diversity and graduality of Ochotona curzoniae images are considered. The interference of isolated background pixels on the clustering result is reduced by mean filtering, with the local Heaviside function being used to replace the original Heaviside function to reduce the search range of the proposed model and suppress the influence of the background on the segmentation results, thereby improving the segmentation performance of the proposed model.

For the purpose of quantitatively analyzing the segmentation performance of the proposed model, the dice similarity coefficient (DSC), Jaccard Similarity (JS) and segmentation accuracy (SA) were used to contrast and analyze the segmentation performance of the proposed model with the other six models [20], as denoted in the following equations:

$$
\begin{aligned}
f_{\mathrm{DSC}} & =\frac{2|\mathbf{A} \cap \mathbf{B}|}{|\mathbf{A}|+|\mathbf{B}|}, \\
f_{\mathrm{JS}} & =\frac{|\mathbf{A} \cap \mathbf{B}|}{|\mathbf{A} \cup \mathbf{B}|}, \\
f_{\mathrm{SA}} & =\frac{\mathrm{TP}+\mathrm{TN}}{\mathrm{TP}+\mathrm{TN}+\mathrm{FP}+\mathrm{FN}},
\end{aligned}
$$

where $\mathbf{A}$ is the ground truth images, as shown in the last row of Figure 1, B is the binary images of algorithm segmentation. TP, TN, FP, and FN are the region-based true positive, true negative, false positive, and false negative, and the example diagram of augmentation accuracy is shown in Figure 2. The larger indicators above indicate that the segmentation result is more accurate.

In Figure 2, the image (a) is an example diagram of FN, $\mathrm{FP}, \mathrm{TP}$, and TN; the image (b) is the FN, FP, TP, and TN on the real image. In the image (a), the black area is TP, the left slash area is FP, the right slash area is FN, and the white area is TN. Correspondingly, in the image (b), the area inside the red curve and yellow curve is TP, the area inside the red curve and outside the yellow curve is FP, the area inside the yellow curve and outside the red curve is FN, and the area outside the red curve and yellow curve is $\mathrm{TN}$.

Through equations (18) and (19), the values of DSC and JS of the images a-f in Figure 1 were calculated. The DSC and JS of the proposed model are shown in Figure 3, where the asterisks represent the result of the proposed model.

From Figure 3, the values of DSC and JS of the model proposed were significantly better than the CV model, LBF model, LIF model, LGIF model, Ding's model, and Zhang's model, which indicates that compared with the other six models, the proposed model can locate the target edge more accurately, and thus the proposed model has a better segmentation effect.

By equation (20), the values of SA of the image a-f in Figure 1 were calculated, as shown in Table 1. In Table 1, the bold values are the optimal segmentation results.

An observation can be made from Table 1 that the accuracy of the proposed model was substantially better than the CV model, LBF model, LIF model, LGIF model, and Ding's model. Further, compared with Zhang's model, the proposed model also offered better results. Multicolor gradual images have inhomogeneous characteristics, and thus the CV model will divide the background of the image into foreground objects by mistake, resulting in a decrease of segmentation accuracy. The LBF model and LIF model can successfully segment the inhomogeneous image by using the 
Step 1. Input image, and set the value of the parameters $c, \lambda_{1}, \lambda_{2}$, and $\mu$.

Step 2. Give the initial contour.

Step 3. Calculate the clustering center of the internal initial contour by fuzzy $C$-means clustering. Step 4. Calculate $c_{2 i}$ and $c_{1}^{\prime}$ by equations (5) and (13).

Step 5. Update the energy function of the CV model by equations (16) and (17).

Step 6. Judge whether the convergence criterion is satisfied or not; if not, go to Step 4.

Algorithm 1: Algorithm flow of the proposed model.

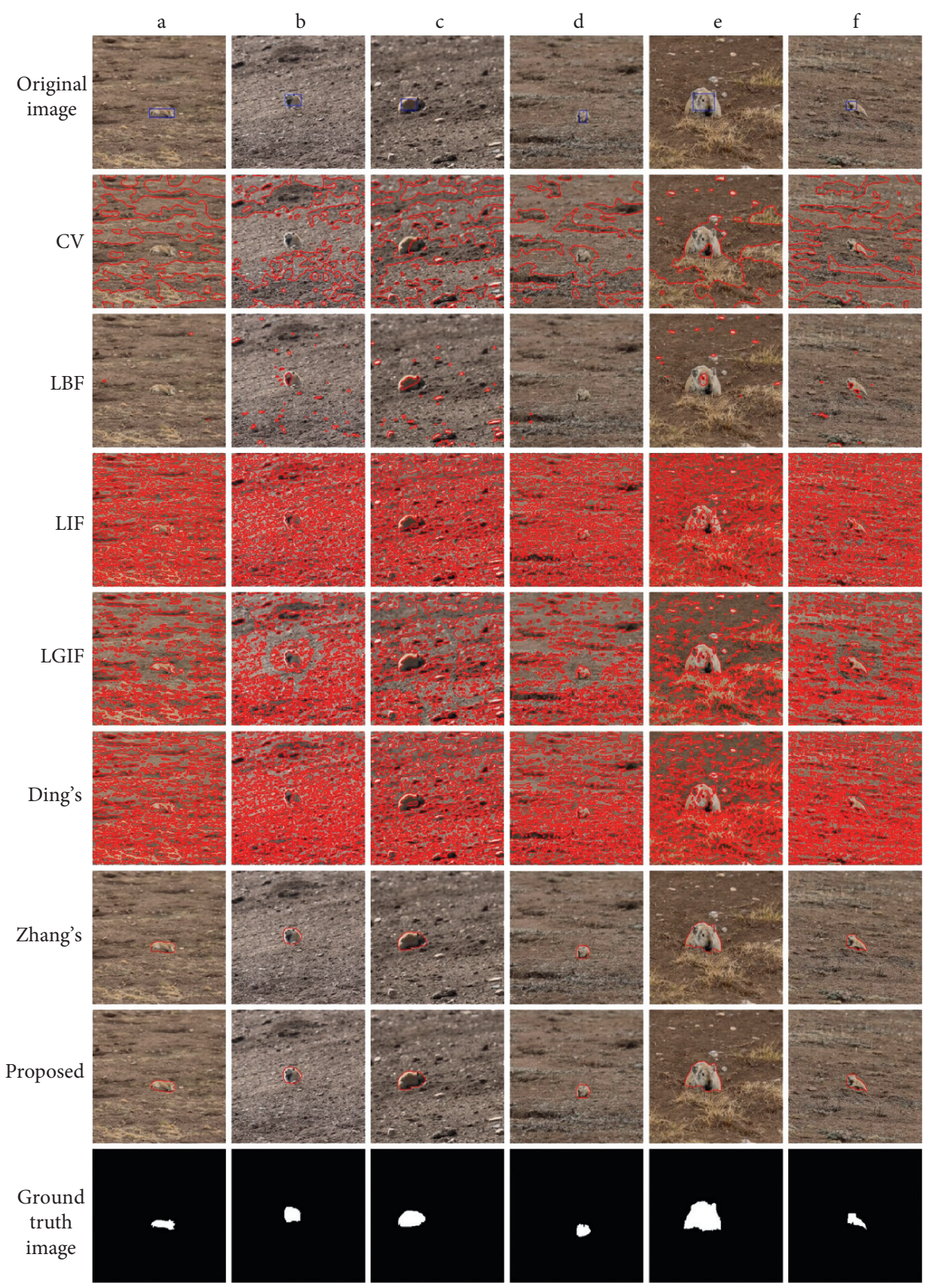

FIGURE 1: Ochotona curzoniae images, segmentation results, and ground truth images. 


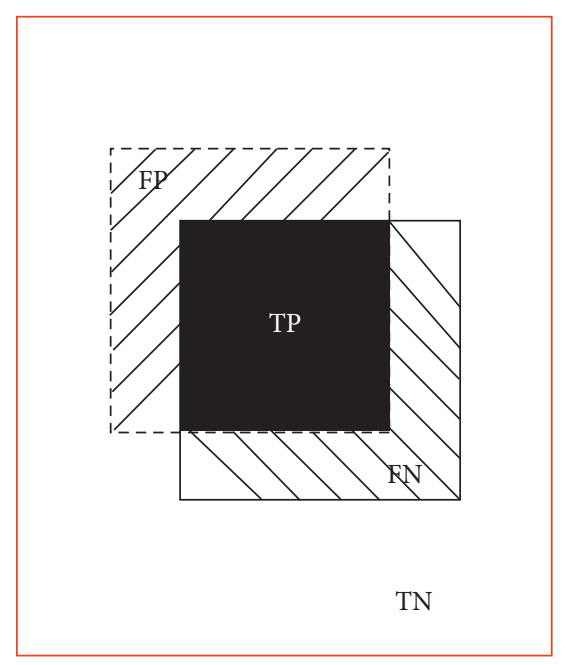

(a)

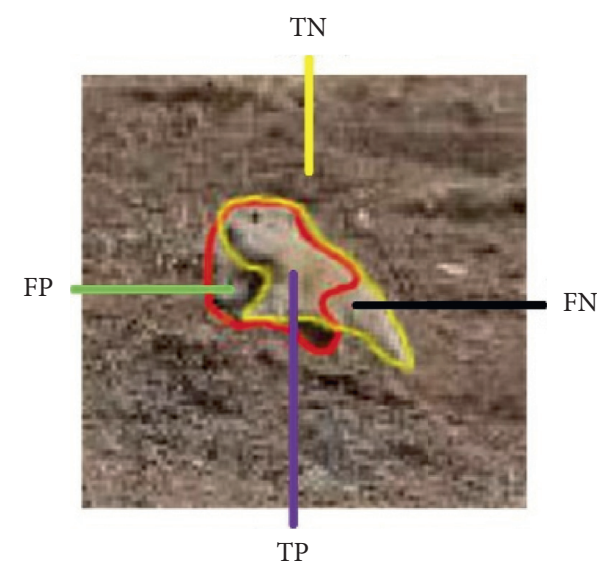

(b)

FIgURE 2: The example diagram of augmentation accuracy. (a) The example diagram of FN, FP, TP, and TN. (b) The FN, FP, TP, and TN on the real image.

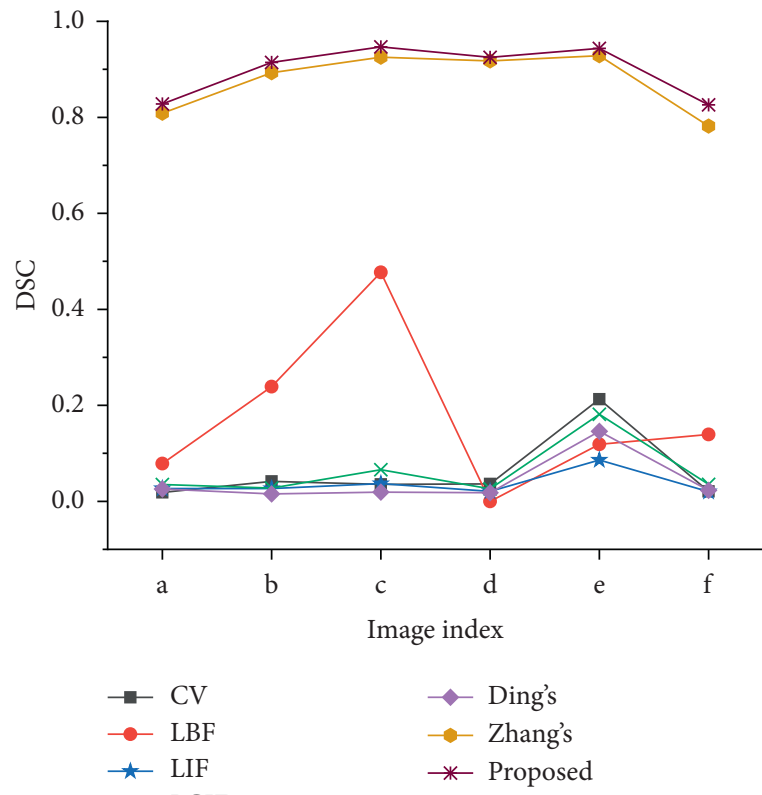

(a)

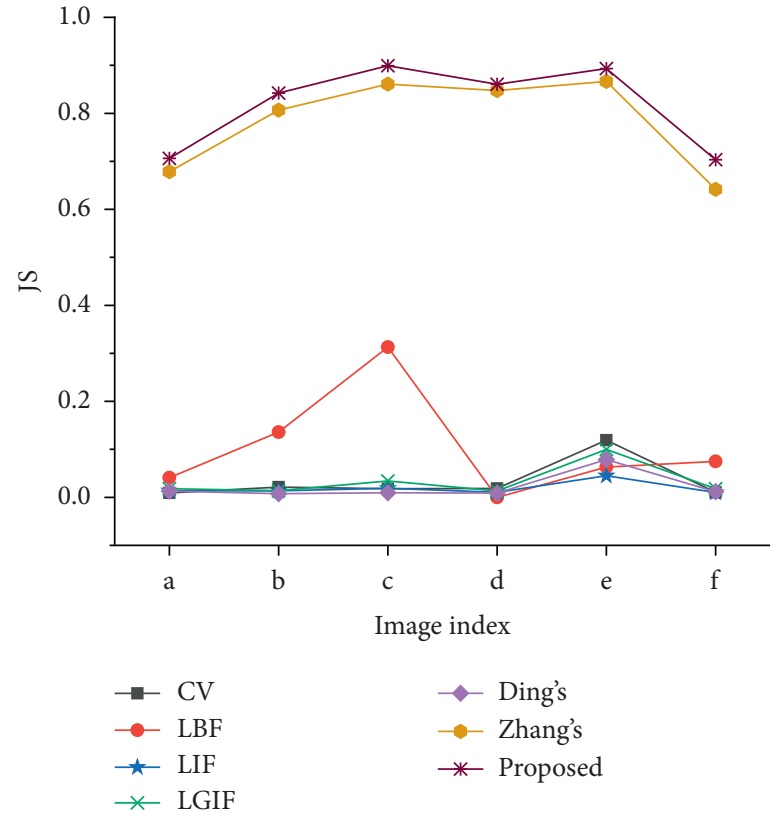

(b)

FIgUre 3: The DSC and JS of the images a-f. (a)The values of DSC. (b) The values of JS.

TABle 1: The SA of the images a-f.

\begin{tabular}{lccccccc}
\hline & CV & LBF & LIF & LGIF & Ding's & Zhang's & Proposed \\
\hline Image a & 0.4792 & 0.9907 & 0.5051 & 0.6255 & 0.4776 & 0.9558 & $\mathbf{0 . 9 9 6 3}$ \\
Image b & 0.4572 & 0.9792 & 0.4947 & 0.4107 & 0.4456 & 0.9973 & $\mathbf{0 . 9 9 7 9}$ \\
Imgae c & 0.4116 & 0.9742 & 0.4971 & 0.5786 & 0.4760 & 0.9969 & $\mathbf{0 . 9 9 7 8}$ \\
Image d & 0.5918 & 0.9917 & 0.4936 & 0.6940 & 0.4428 & 0.9987 & $\mathbf{0 . 9 9 8 8}$ \\
Image e & 0.7021 & 0.9517 & 0.5132 & 0.7270 & 0.6369 & 0.9938 & $\mathbf{0 . 9 9 5 0}$ \\
Image f & 0.5118 & 0.9878 & 0.4887 & 0.6612 & 0.4592 & 0.9955 \\
\hline
\end{tabular}


TABLE 2: The iterations (Iter.) and computation time (CT) of the images a-f in each model.

\begin{tabular}{|c|c|c|c|c|c|c|c|c|}
\hline & & $\mathrm{CV}$ & LBF & LIF & LGIF & Ding's & Zhang's & Proposed \\
\hline \multirow{2}{*}{ Image a } & Iter. & 200 & 180 & 200 & 100 & 100 & 100 & 180 \\
\hline & $\mathrm{CT}(\mathrm{s})$ & 14.31 & 4.662 & 1.537 & 6.586 & 3.225 & 2.317 & 3.180 \\
\hline \multirow{2}{*}{ Image $b$} & Iter. & 70 & 60 & 120 & 220 & 220 & 40 & 60 \\
\hline & $\mathrm{CT}(\mathrm{s})$ & 5.397 & 2.02 & 1.111 & 12.676 & 5.975 & 1.657 & 1.738 \\
\hline \multirow{2}{*}{ Image $c$} & Iter. & 200 & 100 & 200 & 80 & 80 & 180 & 100 \\
\hline & $\mathrm{CT}(\mathrm{s})$ & 14.341 & 2.891 & 1.628 & 5.559 & 2.578 & 3.381 & 2.249 \\
\hline \multirow{2}{*}{ Image d } & Iter. & 180 & 120 & 500 & 40 & 40 & 60 & 120 \\
\hline & $\mathrm{CT}(\mathrm{s})$ & 12.761 & 3.279 & 3.032 & 3.602 & 1.655 & 1.883 & 2.115 \\
\hline \multirow{2}{*}{ Image e } & Iter. & 260 & 160 & 500 & 40 & 40 & 100 & 160 \\
\hline & $\mathrm{CT}(\mathrm{s})$ & 17.852 & 4.199 & 3.272 & 3.618 & 1.705 & 2.288 & 2.996 \\
\hline \multirow{2}{*}{ Image $\mathrm{f}$} & Iter. & 200 & 180 & 200 & 40 & 40 & 140 & 180 \\
\hline & $\mathrm{CT}(\mathrm{s})$ & 14.22 & 4.711 & 1.568 & 3.649 & 1.692 & 2.650 & 3.155 \\
\hline
\end{tabular}

local information of the image, but they can easily fall into a local optimum, and form many small regions, which reduces the segmentation accuracy. The LGIF model can segment the inhomogeneous image by using global and local information of an image, but cannot detect the color gradual feature, thereof leading to poor segmentation. Ding's model used optimized Laplacian of Gaussian to enhance the target edge, but the background of image will also be enhanced, and thus the background will be mistakenly segmented as the target, resulting in significant oversegmentation. Zhang's model used $k$-means to capture the mutagenicity of image, but cannot acquire the graduality of an image, thereby being unable to locate the target edge correctly. In the proposed model, fuzzy $C$-means clustering is used for catching the graduality of an image to segment multicolor gradual images, in addition to using the local Heaviside function, so as to reduce the the influence of the background on segmentation results.

According to Figure 3 and Table 1, the proposed model can effectively segment multicolor images, while due to consideration of the the feature of color gradient, the proposed model has a better adaptive ability to the color diversity of Ochotona curzoniae images. By limiting the search range of the proposed model through the local Heaviside function, the proposed model is more robust against background suppression.

Furthermore, the iterations and computation time of the images $\mathrm{a}-\mathrm{f}$ in each model were compared, as shown in Table 2 .

From Table 2, the iterative times of the CV model were higher than the proposed model, so the computation time of the CV model was longer. The iterative times of the LBF model were the same as the proposed model, but the computation time of the proposed model was han the LBF model. The iterative times of the LIF model were more than the proposed model, but the computation time of the LIF was shorter than the proposed model, which could be attributed to the LIF model using local information for image segmentation and reducing the evolution range of curve. Compared with the proposed model, the iterative times and the computation times of the LGIF model and Ding's model were lower and shorter, respectively, but the proposed model had a higher segmentation accuracy than the LIF model and the LGIF model. The iterative times and computation time of the
Zhang model were lower and shorter, respectively, than the proposed model, which was because $k$-means is faster than fuzzy $C$-means clustering. However, $k$-means cannot capture the gradual feature of images, so the segmentation accuracy of Zhang's model was not as high as the proposed model.

From the experimental results in Figure 3 and Tables 1 and 2, an observation can be made that the model can locate the target edge in the multicolor gradient image more accurately with a low time demand.

\section{Conclusion}

Accurate segmentation of multicolor images is vital to image analysis. Multicolor images have characteristics of color graduality, while the traditional level set models have poor segmentation accuracy. To address this issue, the proposed model uses fuzzy $C$-means clustering to cluster the pixels inside the evolution curve of the CV model, and several clustering centers are obtained to reflect the characteristics of image color diversity. To wholly take into account the gradient of the image color, pixels are divided into different color clusters with a certain probability by fuzzy $C$-means clustering. The clustering center values are used as the inner fitting values of the $\mathrm{CV}$ model, and the images after mean filtering are used to reduce the influence of isolated background points. Further, the global Heaviside function is replaced by the local Heaviside function to suppress the effect of background on the target segmentation. The experimental results on the Ochotona curzoniae image indicate that the proposed model can accurately locate the target edge and has a higher DSC, JS, and ACC; thus, more details in images can be retained.

The proposed model was applied in experiments on Ochotona curzoniae images but can also segment images with diversity and graduality of target colors. In the present study, the feature of strong noise was not considered for the proposed model. Hence, the next step is to segment strong noise images based on the existing methods.

\section{Data Availability}

The experimental data can be obtained from https://github. com/Tust-self/CFCCV. 


\section{Conflicts of Interest}

The authors declare that they have no conflicts of interest regarding the publication of this paper.

\section{Acknowledgments}

This work was supported by the National Natural Science Foundation of China (nos. 61362034 and 62061024).

\section{References}

[1] C. L. Chowdhary and D. Acharjya, "Segmentation of mammograms using a novel intuitionistic possibilistic fuzzy C-mean clustering algorithm," Nature Inspired Computing. Advances in Intelligent Systems and Computing, vol. 652, pp. 75-82, 2018.

[2] H. Liang, H. Jia, Z. Xing, J. Ma, and X. Peng, "Modified grasshopper algorithm-based multilevel thresholding for color image segmentation," IEEE Access, vol. 7, pp. 1125811295, 2019.

[3] Z. Zhang, C. Duan, T. Lin, S. Zhou, Y. Wang, and X. Gao, "GVFOM: a novel external force for active contour based image segmentation," Information Sciences, vol. 506, pp. 1-18, 2020.

[4] B. Chen, S. Huang, Z. Liang, W. Chen, and B. Pan, "A fractional order derivative based active contour model for inhomogeneous image segmentation," Applied Mathematical Modelling, vol. 65, pp. 120-136, 2019.

[5] T. F. Chan and L. A. Vese, "Active contours without edges," IEEE Transactions on Image Processing, vol. 10, no. 2, pp. 266-277, 2001.

[6] C. Li, C.-Y. Kao, J. C. Gore et al., "Implicit active contours driven by local binary fitting energy," in Proceedings of IEEE Computer Society Conference on Computer Vision and Pattern Recognition, pp. 1-7, Minneapolis, MN, USA, June 2007.

[7] K. Zhang, H. Song, and L. Zhang, "Active contours driven by local image fitting energy," Pattern Recognition, vol. 43, no. 4, pp. 1199-1206, 2010.

[8] D. Ma, Q. Liao, Z. Chen, R. Liao, and H. Ma, “Adaptive localfitting-based active contour model for medical image segmentation," Signal Processing: Image Communication, vol. 76, pp. 201-213, 2019.

[9] L. Wang, L. Zhang, X. Yang, P. Yi, and H. Chen, "Level set based segmentation using local fitted images and inhomogeneity entropy," Signal Processing, vol. 167, p. 107297, 2020.

[10] M. Braiki, A. Benzinou, K. Nasreddine, and N. Hymery, "Automatic human dendritic cells segmentation using K-means clustering and chan-vese active contour model," Computer Methods and Programs in Biomedicine, vol. 195, p. 105520, 2020.

[11] N. Memari, A. R. Ramli, M. I. B. Saripan, S. Mashohor, and M. Moghbel, "Retinal blood vessel segmentation by using matched filtering and fuzzy C-means clustering with integrated level set method for diabetic retinopathy assessment," Journal of Medical and Biological Engineering, vol. 39, no. 5, pp. 713-731, 2018.

[12] R. Jin and G. Weng, "Active contour model based on improved fuzzy c-means algorithm and adaptive functions," Computers \& Mathematics with Applications, vol. 78, no. 11, pp. 3678-3691, 2019.

[13] B. Dong, R. Jin, and G. Weng, "Active contour model based on local bias field estimation for image segmentation," Signal Processing: Image Communication, vol. 78, pp. 187-199, 2019.
[14] A. Zhang, F. Wang, and H. Chen, "Multi-color object image segmentation based on improved CV model," Journal of Huazhong University of ence \& Technology, vol. 46, no. 1, pp. 63-66, 2018.

[15] M. Alruwaili, M. H. Siddiqi, and M. A. Javed, “A robust clustering algorithm using spatial fuzzy C-means for brain MR images," Egyptian Informatics Journal, vol. 21, no. 1, pp. 51-66, 2020.

[16] P. D. Pantula, S. S. Miriyala, and K. Mitra, "An evolutionary neuro-fuzzy C-means clustering technique," Engineering Applications of Artificial Intelligence, vol. 89, p. 103435, 2020.

[17] C. L. Chowdhary and D. P. Acharjya, "Clustering algorithm in possibilistic exponential fuzzy C-mean segmenting medical images," Journal of Biomimetics, Biomaterials and Biomedical Engineering, vol. 30, pp. 12-23, 2017.

[18] L. Wang, C. Li, Q. Sun, D. Xia, and C.-Y. Kao, "Active contours driven by local and global intensity fitting energy with application to brain MR image segmentation," Computerized Medical Imaging and Graphics, vol. 33, no. 7, pp. 520-531, 2009.

[19] K. Ding, L. Xiao, and G. Weng, "Active contours driven by region-scalable fitting and optimized Laplacian of Gaussian energy for image segmentation," Signal Processing, vol. 134, pp. 224-233, 2017.

[20] N. Karunanayake, P. Aimmanee, W. Lohitvisate, and S. S. Makhanov, "Particle method for segmentation of breast tumors in ultrasound images," Mathematics and Computers in Simulation, vol. 170, pp. 257-284, 2020. 